\title{
Highly Specialized Optical Metrology
}

\author{
Tadeusz Pustelny* \\ Department of Optoelectronics, Silesian University of Technology, 2A Akademicka St., 44-100 Gliwice, \\ Poland
}

Received December 30, 2014; accepted December 31, 2014; published December 31, 2014

\begin{abstract}
This editorial contains an overview of the contents of the current issue of the Photonics Letters of Poland, devoted mainly to optical metrology. A series of 14 papers deals with both technology as well as pure and applied physics.
\end{abstract}

The scientific topic of the Photonic Letters of Poland, vol. 6(4)/2014 pertains measurements and characterization of photonic structures and systems as well as optical waveguides and nanolayers.

The common denominator of all articles qualified for publication is widely understood, highly specialized optical metrology.

The current issue of PLP presents investigations carried out in selected Polish scientific and research centers.

The group from the Institute of Electron Technology in Warsaw has presented very recent results of investigations into quantum cascade lasers - highly technologically advanced systems.

A paper of the group from West Pomeranian University of Technology in Szczecin is devoted to analyses and investigations of quantum wells structures

The authors from the Department of Optoelectronics at the Silesian University of Technology (SUT) in Gliwice present in several papers analyses, studies and researches concerning integrated optics structures including: interference, multimode interference, structures and systems of differential interferometry. The group from this Department also shows researches of topography and morphology of graphene and graphene oxide with respect to their application in optical and electrical sensors.

The current issue contains also the work, in which the results of investigations are presented into optical fibers in terms of their application in optical telecommunications carried out by the Group from the Faculty of Electronics at the Wroclaw University of Technology.

Birefringence measurements of polymer photonic crystal fibers infiltrated with liquid crystals by using the effect of light depolarization are presented by the authors from the Faculty of Physics at the Warsaw University of Technology.

\footnotetext{
${ }^{*}$ E-mail: Tadeusz.Pustelny@polsl.pl
}

In the paper entitled "A low temperature operated NO2 gas POF sensor based on conducting graft polymer" from the Department of Optoelectronics at SUT in Gliwice novel results are presented of investigations concerning a high sensitive gas sensor based on a polymer optical fiber.

Polymer optical fibers are also the topic of the paper elaborated by a group of the Optical Fiber Technology Laboratory at the University of Lublin. The Group has examined the impact of electromagnetic radiation of high energy on mechanical and optical properties of polymeric materials for their application in the production of optical fibers.

A group from the Gdansk University of Technology has presented the possibility of using polarization sensitive optical coherence tomography in the inspection of ceramic materials.

A group from the Department of Metrology and Optoelectronics at the Poznan University of Technology presents a new approach to objective evaluation of human visual acuity.

Another group from the Faculty of Physics at the Warsaw University of Technology shows the results of their studies on the influence of pitch and birefringence on solitons propagation at the disclination lines in a wedgeshaped planarly oriented sample of the chiral nematic liquid crystal.

A very interesting subject concerning the application of a multi-camera 3D Digital Image Correlation (DIC) system with the procedure of spatial data stitching is presented by the group from the Faculty of Mechatronics at the Warsaw University of Technology. The system diversifies single DIC setup capabilities used for displacement and strain measurements of big and complex structures such as industrial installations.

It should be recognized that the papers contained in the volume 6(4)/2014 are interesting, and the investigations presented in the articles will be continued. 